\title{
Resource Optimization in a Fashion Firm: A Goal Programming Approach
}

\author{
Eronmose Endurance Ajayi-Daniels \\ Department of Applied Information Technology, College of Engineering and Science, Victoria University, Melbourne, Australia
}

\section{Email address:}

eronmose.ajayidaniels@live.vu.edu.au

\section{To cite this article:}

Eronmose Endurance Ajayi-Daniels. Resource Optimization in a Fashion Firm: A Goal Programming Approach. International Journal of Management and Fuzzy Systems. Vol. 5, No. 1, 2019, pp. 14-20. doi: 10.11648/j.ijmfs.20190501.13

Received: December 27, 2018; Accepted: January 31, 2019; Published: February 28, 2019

\begin{abstract}
This study adopted a multi-objective optimization model for a fashion firm using goal programming approach. This was done by optimizing several conflicting objectives at once by using an optimization tool in Linear Programming Solver (LiPs) software. The research objective of optimizing resources in a fashion firm was achieved through the utilization of goal programming whereby goals were prioritized according to importance. The study had three priority level goals and for each priority goal optimum solution was provided. The findings showed that for priority level one the avoidance of overtime, 8 hours is the optimum time as against the 10 hours working time; priority level two showed that a profit of $¥ 8,000$ is realizable as earlier targeted and priority level three showed that the firm can meet its set goal of 3 garments daily. The study concludes that with efficient use of resources, set goals can be achieved.
\end{abstract}

Keywords: Fashion, Garment, Goal Programming, Model, Optimization

\section{Introduction}

There has been a significant change at different levels of the society with respect to technological development, customer's expectation, service delivery, dynamism and complexities of business environment, among others [1]. Due to these changes in the business environment, some organizations go into extinction because of inability to cope and survive. Some others have gone through the thick and thin still surviving and growing. The challenges facing fashion industry in Nigeria called for the adoption of multiple goals and objectives by striving to produce the best quality output at a profit or at the lowest minimum cost.

In achieving this, effective production planning is required. At every stage of creating an output, careful planning is required that must be in line with the multiple goals of the organization. Due to the possibility of having conflicting goals, proper planning and effective implementation must be put in place to optimize organizational resources such as capital, labour, time, among others in achieving set goals. [2] asserted that the activities of planning aim at attaining the long term goals through the short term goals. For a fashion firm, attaining its long term goals requires achieving its monthly or quarterly goals.
The fashion industry in Nigeria is fast growing and it has become a main source of livelihood for many families. At the individual level, it serves as subsistence output for the family, at the organization level, it boost the sales and profit of the organization, while at the national level, it creates not just employment but also boost the GDP of the country. The demand for fashion products in Nigeria is increasing thereby making the industry to become more competitive than ever before [3]. For this reasons, fashion designers are faced with multiple criteria decision challenges that impact on their viability and profitability in the market place. The multiple criteria that have to be considered include trying to optimize profit margin and ensuring the efficient use of the resources at their disposal in an environment that is characterized with infrastructural deficit, increasing market competition, stiffer government regulations and increasing prices of resource inputs. The management of fashion firms are thus faced with the challenge of how to satisfy all these goals simultaneously.

Goal programming as an operations research technique has been found useful in achieving multiple goals with constrained resources. Examples of its application in many manufacturing industries include bakery industry [4-6], rubber/wood door manufacturing factory [7]; toothpaste factory [8], among others. Review of extant literature has 
shown that less attention is given to the application of goal programming in the fashion industry especially in the Nigerian context. It is against this backdrop that this study examined the utilization of goal programming technique of the multi criteria decision making to determine an optimal solution that satisfies the multi-objective based decisions of the management of Luciano Fashion Outfit in Benin City, Edo State, Nigeria.

\section{Literature Review}

\subsection{Concept of Goal Programming}

Goal programming (GP) is a branch of multi-objective optimization, which in turn is a branch of multi-criteria decision analysis (MCDA), also known as multiple-criteria decision making (MCDM). Goal programming is an optimization programme. It can be thought of as an extension or generalization of linear programming to handle multiple, normally conflicting objective measures [9]. Each of these measures is given a goal or target value to be achieved. Unwanted deviations from this set of target values are then minimized in an achievement function. This can be a vector or a weighted sum dependent on the goal programming variant used. Goal programming is used to perform three types of analysis which basically is the objective of this study with respect to the fashion firm: determining the required resources to achieve a desired set of objectives; determining the degree of attainment of the goals with the available resources; and providing the best satisfying solution under a varying amount of resources and priorities of the goals.

Goal programming underlies a realistic satisficing philosophy. Coherently, with the satisficing philosophy in the GP models the deviations between the achievement of the goals and their targets are minimized, that is, a certain function of the unwanted deviation variables is minimized [10]. According to Orumie [9], the concept of goal programming has been advanced by some theorists such as Charnes and Cooper, Ferguson, Lee, Ignizio, among others. Prior to when it was founded by Charnes [11], there was no goal programming and not until the middle of the 1970s, GP applications reported in the literature were rather scarce. Since that time and chiefly due to seminal works by Lee and Ignizio, an impressive boom of GP applications and technical improvements have arisen. It can be said that GP has been, and still is, the most widely used multi-criteria decision making technique. Basically, GP is designed to minimize the unfavorable deviations from purposes and it is applicable to multiple and conflicting goals problems.

Goal programming may be used to solve linear programs with multiple objectives, with each objective viewed as a "goal". In goal programming, $\mathrm{d} i+$ and $\mathrm{d} i$-, deviation variables, are the amounts a targeted goal $i$ is overachieved or underachieved, respectively. The goals themselves are added to the constraint set with $\mathrm{d} i+$ and $\mathrm{d} i$ - acting as the surplus and slack variables [12]. One approach to goal programming is to satisfy goals in a priority sequence. Second-priority goals are pursued without reducing the first-priority goals, and so on. For each priority level, the objective function is to minimize the (weighted) sum of the goal deviations. The situation whereby goals are prioritized is referred to as preemptive goal programming. Such a situation arises when one or more of the goals clearly are far more important than the others. Thus, the initial focus should be on achieving as closely as possible these first-priority goals. The other goals also might naturally divide further into second-priority goals, thirdpriority goals, and so on. After finding an optimal solution with respect to the first-priority goals, we can break any ties for the optimal solution by considering the second-priority goals. Any ties that remain after this re-optimization can be broken by considering the third-priority goals, and so on.

Goal programming models can be classified in terms of the mathematical nature of the decision variables and goals introduced. These include fuzzy, integer, binary and fractional goal programming. Goal programming can also be classified into different variants in terms of their underlying distance metric and philosophy used. These include the lexicographic/pre-emptive, weighted/non- pre-emptive and Chebyshev goal programming variants [13].

\subsection{Goal Programming Approaches}

The idea of goal programming is to convert original multiple objectives into a single goal. The resulting model yields a satisficing solution which may not be optimum with respect to all the conflicting objectives of the problem. That is, "GP yields only an efficient and satisfactory result rather than optimum, solution to the problem. This is because, it is uncommon to always satisfy every goal, so goal programming attempts to reach a satisfactory level of the multiple objectives under consideration [11]. To avoid the possible bias effect of the solution to different measurement unit, goal normalization takes place. The procedures for structuring goal programming model are similar to those for a linear programming. The main difference between the LP and GP is that, LP maximizes or minimize (optimizes) a single objective functions whereas, GP minimizes the deviations between the target values of the objectives and the realized results (satisficing solution). The basic steps for structuring goal programming are as follows [14]:

a. Goals are discovered and converted to constraints by introducing deviational variables.

b. Examine the goals to determine the exact deviational variables needed for them, that is, whether $d_{i}^{-}, d_{i}^{+}$, or both.

c. In the second objective goal, it implies that anything below the target value $b_{i}$ is acceptable, so the overachievement of the target $d_{i}^{+}$should be minimized to 0 . In row three, the objective goal is that anything below the target value $b_{i}$ should be driven to zero while the over-achievement of the target $d_{i}^{+}$should be accepted. The last objective goal implies that anything below or above the target value $b_{i}$ is unacceptable, so the over-achievement of the target $d_{i}^{+}$and under achievement of the goal $d_{i}^{-}$should be minimized to 0 .

d. Goals are ranked in order of importance and preemptive priority factor. 
e. In case of ties in priority, assign weights to each of the deviational variables in the priority.

Once the above steps are completed, the problem can be quantified as a GP model. The approaches or steps can simply be:

Step 1: Decide the priority level of each goal.

Step 2: Decide the weight on each goal.

If a priority level has more than one goal, for each goal $i$ decide the weight, $w_{i}$, to be placed on the deviation(s), $d_{i}^{+}$and/or $d_{\bar{i}}$, from the goal.

Step 3: Set up a linear program. Consider new objectives (minimize deviations), subject to all functional constraints, and goal constraints.

Step 4: Solve the current linear program."

\subsection{Goal Programming Assumptions and General Principles}

The "axioms of goal programming models as follows [15]: a. Additivity: This implies that the level of penalization for undesired deviational variables from a target level does not depend on the levels of unwanted deviational variables from the other goals.

b. Proportionality: This assumption in the goal programming model requires that the penalization for an unwanted deviational variable from a target level is directly proportional to the distance away from the target level.

c. Divisibility: This assumption implies that all the decision variables should be free to take any value within their stated range, that is, a decision variable cannot be forced to take an integer or a discrete value.

d. Certainty: This assumption implies that all the data coefficients are known with certainty. However, the use of goal programming is not necessarily impossible if any of the above axioms is violated. A nonlinear goal programme could be formulated if the additive condition does not hold. In the case where the divisibility axiom does not hold, an integer or binary goal programming could be formulated. When the certainty axiom is not holding, then the method to be used will depend on the type of coefficients over which there exists uncertainty. A certain amount of uncertainty over weights and target values often exists and this can frequently be handled by good sensitivity or weight analysis techniques or an interactive method. Another good alternative is to use the fuzzy goal programming variant. If there is uncertainty over the technological coefficients then either the fuzzy goal programming variant or a combination with a simulation technique could be used.

e. Satisficing: Goal programming is primarily a satisficing technique. Satisficing is a behaviour in which decision makers aim to reach a set of defined goals [16]. If they reach those goals, then they are satisfied. This is different from the concept of optimizing. Human beings are more interested and able to reach goals than in the abstract concept of optimizing each outcome of the decision problem [16]. Meeting goals as closely as possible is the main aim of the goal programming technique. Satisficing as the prime underlying philosophy of goal programming and that its solutions should be judged solely on how well they meet the goals of the decision maker and whether they produce a practical solution to the decision problem [13]. Although goal programming can produce Pareto-inefficient solutions, this is mainly due to poor formulation and modeling of the decision maker's preferences and target levels by the analyst building the goal programme.

f. Optimizing: Optimization implies looking for the decision which gives the best possible value of some measure of performance from amongst the set of possible decisions. The theory of optimizing in the presence of multiple objectives is defined by adapting a concept of Pareto optimality in a multiobjective model. Optimizing philosophy has importance in goal programming in the situations where: if Pareto optimality detection and restoration take place then the goal programme has a mix of the satisficing and optimizing philosophies; and if the goals are two-sided (i.e. a particular value is optimal rather than a "more is better" or "less is better" situation) then the satisficing and optimizing philosophies can be thought of as coinciding for those goals [13].

g. Ordering or Ranking: Ordering or ranking is important in the lexicographic goal programming because, it is assumed that the ranking of the goals in order of importance to the decision maker exists and is known or able to be estimated by the decision maker. In real-life situations, goals does not take place lexicographically, and in these cases the decision maker will explore the trade-offs or the balance between the goals." In these cases lexicographic goal programming should not be used and instead another goal programming variant should be chosen.

\subsection{Review of Previous Studies}

Many studies have been conducted on optimization of resources in different firms, with more focused on financial industry, agriculture, bakery and other related manufacturing firms. Some of the "studies that used goal programming especially in the Nigerian context are reviewed as follows:

Goal programming model was employed for production planning in toothpaste factory, with two priority structures of exploring the trade-off options between two objectives of minimizing processing cost, and maximizing the capacity utilization of production facilities [8]. The study revealed that when cost minimization was given first priority, the total processing cost (normalized) was achieved by $\$ 247,678$ based on the value of the deviational variable $1=0+d$, while the utilizations of Processing Plant 1 and Filling Machine 2 were $20.32 \%$ and $0.18 \%$ respectively, thus indicated serious under-utilization of PP1 and FM2 which are almost idle. The study found that the cost goal was achieved but the capacity utilization of production facilities was underachieved by $8.2 \%$ when cost minimization was given first priority, and attributed the capacity under utilization to the design of the production system which appears to far below optimal, technically unhealthy and economic inefficiency of the facility and the production system as a whole. The study further revealed that when capacity utilization of production facilities was assigned first priority, 
the capacity utilization goal was achieved or improve by $1=$ $18689+\mathrm{d}$, and which indicated that cost goal was also overachieved by $\$ 18,689$, which translated into an increase of $7.55 \%$ in the processing cost. The study concluded that the utilization of the least-utilized facility was $43.85 \%$ as against $0.18 \%$ when cost minimization was assigned first priority.

Goal programming was used in Imo State University to examine appropriate budgetary allocation with respect to achieving its five priorities (goals) which are personal cost, overhead cost, capital expenditure, revenue (internally generated) and the total budget) [17]. Based on the report generated, it was found that the optimum value of $Z(Z=4.24)$ satisfied goal 1(the personal cost goal), goal 3 (capital expenditure goal) and goal 5 (the total budget goal), but failed to satisfy goal 2 and goal 4, which are overhead cost and revenue goals respectively. The study suggested that the University should disburse $\$ 4.24$ billion to satisfy goal 2 and goal 4, which are overhead cost and revenue goals respectively.

Multi-criteria feed formulation was used to determine the productivity of Nigerian feed Mill Industry using a mathematical programming in bringing about an optimal solution that is economical in nature [18]. With the alternative optimal solutions factored into the productivity model together with other input factors such as the cost of fuel ( $\mathrm{C}$ fuel), cost of labour ( $\mathrm{C}$ labour) and cost of packaging (C packaging), the study revealed that productivity index estimated from the four alternative optimal formulations were $1.13,1.144,1.06$, and 0.96 respectively, which showed that the optimal feed formulation generated in alternative 2 produces the highest profit $(14.35 \%)$ and hence highest productivity index (1.144).

Linear goal programming technique was applied in Rufus Giwa Polytechnic Bakery, Ondo State, Nigeria to optimize the production of three types of bread: medium bread $\left(\mathrm{X}_{1}\right)$, large bread $\left(\mathrm{X}_{2}\right)$ and extra-large bread $\left(\mathrm{X}_{3}\right)$ linear goal programming technique [5]. Based on the report generated by linear goal programming software version 15 , it was revealed that the extra-large bread shows a significant profit of approximately $\$ 203.6$ based on the mean value of 184.4 for temperature per unit of extra-large bread production as against medium and large bread that shows a profit of $\$ 113.4$ and $\$ 23.6$ based on the mean value of 192.2 and 188.8 for temperature per unit of medium and large bread production respectively. The study therefore indicated that optimal solution was attained at $\mathrm{X}_{3}=1.175$, which is production of extra-large bread $\left(\mathrm{X}_{3}\right)$ at given constraints, while that of medium and large bread were not achieved or not economical to produce as they do not contribute to the maximum profit the institution bakery in the face of the constraints they operate upon." The study recommended that bakery should stop the production of medium and large bread and produce 235pieces of extra-large bread only from 1.175unit (1 bag of flour) per day for them to make a maximum profit of $\$ 47572.28$ (\$239.19) per day or the unit profit on the medium bread and large bread must increase to $\$ 39474.87$ $(\$ 198.48)$ and $\$ 37450.52$ (\$188.30) respectively before it becomes economical to produce.

Based on the above review, goal programming has been widely used in achieving multiple goals with constrained resources in different setting such as bakery industry [4-6], feed formulations [18]; rubber/wood door manufacturing factory [7]; toothpaste factory [8], among others.

\section{Methodology}

The general goal programming model can be expressed mathematically [19]:

Minimize:

$$
Z=\sum_{i=1}^{m} W_{i} P_{i}\left(n_{i}+p_{i}\right)
$$

Subject to:

$$
\sum_{j=1}^{n} a_{i j} x_{j}+n_{i}-p_{i}=b_{i}
$$

For all I and j:

$$
x_{j} n_{i} p_{i} \geq 0
$$

From the above, equation (1) represents the objective function and the variables are defined as:

$Z=$ The sum of all deviations from the desired goals (negative and positive deviations inclusive)

$\mathrm{M}=$ The total number of constraints in the given goal programming problem

$\mathrm{I}=(1,2 \ldots \ldots \ldots)$ the total number of goals in the model

$n_{i}=$ Negative deviation from a relevant goal, $\mathrm{i}$

$p_{i}=$ Priority level assigned to each relevant goal ranked order (i.e $\mathrm{P}_{1}>\mathrm{P}_{2}>\mathrm{P}_{3}$ )

$W_{i}=$ Non negative constants that represent the relative weights to be assigned to the decision variable.

Equation (2) represents the goal constraints of the problem. This expresses the relevant resource limits that may restrict the achievement of the goals.

$a_{i j}=$ Constant attached to each decision variable

$b_{i}=$ The resource values that are considered to be utilized for objectives

$x_{j}=(\mathrm{j}=1,2 \ldots \ldots \ldots \mathrm{m})$ an unknown decision variable that is being determined

Equation (3) expresses the non-negative requirement where $x_{j} n_{i}$ and $p_{i}$ for all $\mathrm{i}$ and $\mathrm{j}$ must be greater than or equal to zero. Also, negative and positive deviation cannot occur at the same time for a particular constraint $i$. Therefore, it can be said that the product of $n_{i}$ and $p_{i}$ is always zero.

The various goals this study attempts to achieve with respect to the determination of the level of achievement of the firm goals as a consideration of multiple criteria decision making are:

i. to produce a minimum amount of garment daily (Goal 1 also known as $\mathrm{G}_{1}$ )

ii. to achieve daily profit of a certain amount (Goal 2 also known as $\mathrm{G}_{2}$ )

iii. to avoid the use of overtime (Goal 3 also known as $\mathrm{G}_{3}$ )

As a pre-emptive goal programming where goals are 
prioritized, it is assumed that $G_{1}>G_{2}>G_{3}$. Also, $X_{1}, X_{2}, X 3$, $\mathrm{X}_{4}$ connote the decisional variables, where:

$\mathrm{X}_{1}=$ amount of thread to make a garment

$\mathrm{X}_{2}=$ amount of button to make a garment

$\mathrm{X}_{3}=$ number of employees assigned to cut a material

$\mathrm{X}_{4}=$ number of employees assigned to sow a material

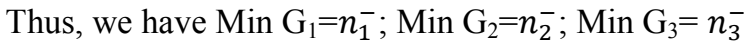

Subject to: $\mathrm{X}_{1}+\mathrm{X}_{2}+\mathrm{X}_{3}+\mathrm{X}_{4}+n_{1}^{-}-p_{1}^{+}=b_{1}$

$\mathrm{X}_{1}+\mathrm{X}_{2}+\mathrm{X}_{3}+\mathrm{X}_{4}+n_{2}^{-}-p_{2}^{+}=b_{2}$

$\mathrm{X}_{1}+\mathrm{X}_{2}+\mathrm{X}_{3}+\mathrm{X}_{4}+n_{3}^{-}-p_{3}^{+}=b_{3}$

$\mathrm{X}_{1}, \mathrm{X}_{2}, \mathrm{X}_{3} \mathrm{X} 4, n_{1}^{-}, p_{1}^{+}, n_{2}^{-}, p_{2}^{+}, n_{3}^{-}, p_{3}^{+} \geq 0$

The justification for using thread and button as well as those who are assigned to cut and sew a material as resources constraints is because of the nature of the firm, which is into the designing and sewing of native attires. Another justification is that these resources beside sewing machine constituted the major factors required by fashion firm to produce a garment.

The data generated for this study were obtained from primary and secondary sources. The data collected through primary source were done by interviewing the manager of the fashion firm. This helps to gain first-hand information about the firm operations, number of employees, time spent and other materials used in their daily operations. Data collected through secondary source were done by asking the manager for the company records.

Data collected were analyzed using the goal programming model algorithm. This was made possible with the use of LiPs software. The goal programming is a popular multiobjective optimization estimation technique used in handling problems with multiple objectives.

\section{Empirical Analysis and Result}

Table 1. Analysis of the variables.

\begin{tabular}{|c|c|c|c|c|}
\hline Variables/Goals & Garment & Profit per item & Time utilized & System constraints \\
\hline Threads $\left(\mathrm{X}_{1}\right)$ & 2 & 12 & Inclusive & 2 \\
\hline Buttons $\left(\mathrm{X}_{2}\right)$ & 3 & 8 & 4 minutes & 6 \\
\hline Employees to cut and sew male garments $\left(\mathrm{X}_{3}\right)$ & 2 & 2,000 & 2 hours & 2 \\
\hline Employees to cut and sew female garments $\left(\mathrm{X}_{4}\right)$ & 2 & 3,000 & 3 hours & 2 \\
\hline
\end{tabular}

Information on the resources with respect to garment, profit and time is provided in Table 1. Also, Table 1 contains here is the system constraints experienced by the fashion firm. The Table further indicates the variables and goals of the firm by specifying their respective resources available.

This section provides us with the following priority level analysis of the above problem. First, the model was specified before the analysis of each goal was done. The analysis of the goals according to importance began with the garment goal, then to the profit goal and then to overtime goal.

From the above analysis as presented

$2 \mathrm{X}_{1}+3 \mathrm{X}_{2}+\mathrm{X}_{3}+\mathrm{X}_{4}+n_{1}^{-}-p_{1}^{+}=5$ (garment goal)

$12 \mathrm{X}_{1}+8 \mathrm{X}_{2}+2 \mathrm{X}_{3}+3 \mathrm{X}_{4}+n_{2}^{-}-p_{2}^{+}=8$ (profit goal)

$\mathrm{X}_{1}+4 \mathrm{X}_{2}+2 \mathrm{X}_{3}+3 \mathrm{X}_{4}+n_{3}^{-}-p_{3}^{+}=10$ (overtime goal)

$\mathrm{X}_{1} \geq 2$ (thread constraint)

$\mathrm{X}_{2} \geq 6$ (button constraint)

$\mathrm{X}_{3} \geq 2$ (Assign at least 2 employees for male garment)

$\mathrm{X}_{4} \geq 2$ (Assign at least 2 employees for female garment)

$\mathrm{X}_{1}, \mathrm{X}_{2}, \mathrm{X}_{3} \mathrm{X} 4, n_{1}^{-}, p_{1}^{+}, n_{2}^{-}, p_{2}^{+}, n_{3}^{-}, p_{3}^{+} \geq 0$

\subsection{Priority Level 1 Program}

There is one goal in priority level one, which is the overtime goal

$\operatorname{Min} \mathrm{G}_{1=} d_{1}^{-}$

Subject to $2 \mathrm{X}_{1}+3 \mathrm{X}_{2}+d_{1}^{-}-d_{1}^{+}=10$

$$
\begin{gathered}
2 \mathrm{X}_{1}+3 \mathrm{X}_{2}+d_{2}^{-}-d_{2}^{+}=8 \\
\mathrm{X}_{1}+\mathrm{X}_{2}+d_{3}^{-}-d_{3}^{+}=3 \\
\mathrm{X}_{1} \geq 2 \\
\mathrm{X}_{2} \geq 2
\end{gathered}
$$

$$
\mathrm{X}, d_{i}^{-}, d_{i}^{+} \geq 0
$$

The optimum solution (determined by LiPS) is $\mathrm{X}_{1}=1$ hour, $\mathrm{X}_{2}=2$ hours, $d_{1}^{-}=2$ (check appendix). The solution shows that the overtime goal, $\mathrm{G}_{1}$ is violated by 2 hours. Therefore, the additional constraint to be used in solving for $G_{2}$ problem is $d_{1}^{-}=2$ (or equivalent, $\leq 2$ ).

\subsection{Priority Level 2 Program}

There is one goal in priority level two, which is the profit goal and the objective function is given below subject to same set of constraints in priority level 1 plus the additional constraint, $d_{1}^{-}=2$. This additional constraint $d_{1}^{-}=2$ can also be accounted for by substituting out $d_{1}^{-}$in the first constraints. The result is that the right hand side of the overtime goal constraint will be reduced from 10 to 8 , thus we have:

$$
\operatorname{Min} \mathrm{G}_{2=} d_{2}^{-}
$$

$$
\begin{gathered}
\text { Subject to } 2 \mathrm{X}_{1}+3 \mathrm{X}_{2}+d_{1}^{-}-d_{1}^{+}=8 \\
\qquad \begin{array}{c}
2 \mathrm{X}_{1}+3 \mathrm{X}_{2}+d_{2}^{-}-d_{2}^{+}=8 \\
\mathrm{X}_{1}+\mathrm{X}_{2}+d_{3}^{-}-d_{3}^{+}=3 \\
\mathrm{X}_{1} \geq 2 \\
\mathrm{X}_{2} \geq 2 \\
\mathrm{X}, d_{i}^{-}, d_{i}^{+} \geq 0
\end{array}
\end{gathered}
$$

The optimum solution (determined by LiPS) is $\mathrm{X}_{1}=1, \mathrm{X}_{2}=$ 2 , with the remaining variables equal to zero. The solution shows that the profit goal, $G_{2}$ is optimum at 8,000 . Therefore, the additional constraint to be used in solving for $\mathrm{G}_{3}$ problem 
is $d_{2}^{-}=8$ (or equivalent, $\leq 8$ ). (indicating no deviation)

\subsection{Priority Level 3 Program}

There is one goal in priority level three, which is the garment goal subject to same set of constraints in priority level 1 plus the additional constraint $d_{2}^{-}=8$

$$
\begin{gathered}
\operatorname{Min}_{3=} d_{3}^{+} \\
\text {Subject to } 2 \mathrm{X}_{1}+3 \mathrm{X}_{2}+d_{1}^{-} d_{1}^{+}=8 \\
2 \mathrm{X}_{1}+3 \mathrm{X}_{2}+d_{2}^{-}-d_{2}^{+}=8 \\
\mathrm{X}_{1}+\mathrm{X}_{2}+d_{3}^{-}-d_{3}^{+}=3 \\
\mathrm{X}_{1} \geq 2 \\
\mathrm{X}_{2} \geq 2 \\
\mathrm{X}, d_{i}^{-}, d_{i}^{+} \geq 0
\end{gathered}
$$

The optimum solution (determined by LiPS) is $\mathrm{X}_{1}=1, \mathrm{X}_{2}=$ 2 , with the remaining variables equal to zero. The solution shows that the garment goal, $\mathrm{G}_{3}$ is optimum at 3 garments. The optimization of $\mathrm{G}_{3}$ is not necessary because the optimum solution to the problem $\mathrm{G}_{2}$ already yields $d_{3}^{-}=0$. Hence, the solution of $\mathrm{GP}_{2}$ is automatically optimum for $\mathrm{GP}_{3}$ as well. The solution $d_{3}^{-}=0$ shows that $\mathrm{G}_{3}$ is satisfied.

\subsection{Discussion of Findings}

The primary aim of this study is to find optimal solution that satisfies the multi-objective based decisions of the management of Luciano fashion Outfit. From the first priority goal, which is avoiding the use of overtime, the report of the LiPs analysis showed that to attain optimality, management needs to spend one hour and two hours on making male garments and female garments respectively. In so doing, the daily time that ought to be spent in making garments is 8 hours. The report indicated that an extra 2 hours is being spent as overtime in producing the same amount of garment. By implication, the management can make optimal use of the available labour time by actually having a repositioning of the business. The result also implies that the $\mathrm{GP}_{1}$ is relevant for time management and business process reengineering. This is in line with previous findings that formulated a strategic planning model using the goal programming approach to increase production quantity, net profit, customers demand and to decrease expenditure and processing times of machines [20]. This outcome is similar previous finding in which the importance of time and planning phase in an activity was emphasized [21].

On priority or goal level two, the objective was to maximize profit. From the analysis, the LiPS report indicated that the optimum solution is reached when the profit is 8,000 which is the same as earlier projected. However, report further showed that this same profit can be achieved by realizing 1, 000 and 2,000 on male and female garments respectively. This goes to show that the management should reconsider the price at which it sells her garments. This model can be said to be relevant to pricing. This supports previous findings that emphasized the use of goal programming to marketing campaigns [22].

The analyses of the third goal showed that optimality is found at 3, that is, management should produce 3 garments daily. However, the report required that the management should produce 1 male garment and 2 female garments daily. This model by implication is relevant to capacity utilization rate policy. This finding is supported by previous findings that showed that the number of products to be produced to achieve the most optimal production results [23].

\section{Conclusion and Recommendations}

The utilization of goal programming models can help firms to reach optimal solutions which allow for appropriate decisions to be reached thereby affecting the organization positively. With goal programming, management can utilize their limited resources to achieve set goals optimally. The application of goal programming to management of a firm in dynamic and complex environment of business cannot be overemphasized as this allows for strategic planning and effective decisions making. This study is relevant to polices on time management, business process reengineering, pricing and capacity utilization rate.

On the basis of the findings of this study, we recommend the followings. First, management of Luciano Fashion Outfit should make use of five workers against three workers working at minimum of 8 hours daily if the organisation is to achieve set goals. Second, management Luciano Fashion Outfit should be engaged more in the production of female garments since it is more profitable. This can be done by using extra workers to produce female garments rather than male garments. Third, Luciano Fashion Outfit should adopt a proper time management plan to ensure that its goals/targets are met within stipulated time.

Finally, further studies on the subject matter can be extended to capture other resources like the number of machines, amount of thread and or button needed to produce a garment. Also, sensitivity analysis can be carried out if the parameters (input data) of the model is changed such as changes in the resources available (right hand side of the constraints) or changes in the objective function.

\section{References}

[1] Arrenofsky, J. S., Dutton, J. M., \& Tayyabkhan, M. T. (2010). Managerial programming with linear programming in process industry operations. New York: John Wiley and Sons Inc.

[2] Maroon, L. (2011). An introduction to lean thinking. Lean Enterprise Research Centre, Cardiff Business School.

[3] Agu, G. A., \& Onuoba, A. O. (2016). Psychological influences and fashion consumption behaviour of consumers in South East Nigeria. International Journal of Research in Business Studies and Management, Vol. 3, No.12, Pp. 56-63. 
[4] Akpan, N. P., \& Iwok, I. A. (2015). Application of linear programming for optimal use of raw materials in bakery. International Journal of Mathematics and Statistics Invention, Vol. 4, No. 8, Pp.51-57.

[5] Raimi, O., \& Adedayo, O. C. (2017). Application of linear programming technique on bread production optimization in Rufus Giwa Polytechnic Bakery, Ondo State, Nigeria. American Journal of Operations Management and Information Systems, Vol. 2, No. 1, Pp. 32-36.

[6] Nasruddin, H., Afifah, H., Nur, S., \& Nurul, F. R. (2013). A goal programming model for bakery production. Advances in Environmental Biology, Vol. 7, No.1, Pp. 187-190.

[7] Sen, N. \& Nandi, M. (2012). An optimal model using goal programming for rubber wood door manufacturing factory in Tripura. Mathematical Theory and Modeling, Vol. 2, No.8, Pp.31-36.

[8] Adeyeye, A. D., \& Charles-Owaba, O. E. (2008). Goal programming model for production planning in a toothpaste factory. South African Journal of Industrial Engineering, Vol. 19, No.2, Pp. 197-209.

[9] Orumie, U. C., \& Ebong, D. W. (2014). An efficient method of solving lexicographic linear goal programming problem. International Journal of Scientific and Research Publications, Vol. 3, No.7, Pp. 1-8.

[10] Agawal, V., \& Kholi, P. (1991). Computer aided robot selection: The multiple attribute making approach. International Journal of Production Research, Vol. 29, No.8, Pp. 1629-1640.

[11] Charnes, A., \& Cooper, W. (1961). Goal programming and multiple objective optimization, European Journal of Operational Research, Vol. 1, No.7, Pp. 39-54.

[12] Belton, V., \& Stewart, T. J. (2002). Multiple criteria decision analysis: An integrated approach. Kluwer: Academic Publishers.

[13] Tamiz, M., Jones, D. F., \& El-darzin, E. (2008). A review of goal programming and its applications. Annals of Operation Research, Vol. 58, No. 1, Pp. 39-53.
[14] Rifai, A. K. (2006). A note on the structure of the goalprogramming model: Assessment and evaluation. International Journal of Operations and Production Management, Vol. 16, No.4, Pp. 40-49.

[15] Winston, W. (2004). Operations research: Applications and algorithms. CA: Duxbury Press.

[16] Simon, H. A. (1977). The logic of heuristic decision making. In Models of discovery (pp. 154-175). Springer, Dordrecht.

[17] Ekezie D. D., \& Onuoha, D. O. (2013). Goal programming: An application to budgetary allocation of an institution of higher learning. Research Journal in Engineering and Applied Sciences, Vol. 2, No.2, Pp. 95-105.

[18] Abayomi, O. O., Temitope, A. K., Oyelayo. O., \& Oluseye, A. A. (2015). A productivity outlook for a multi-criteria animal feed formulation problem: A case study of Nigerian feed Mill Industry. International Journal of Advancements in Technology Research, Issue 6, Pp. 145-153.

[19] Sharma, J. K. (2013). Operations research theory and applications. New Delhi: Macmillan publishers.

[20] Sinha, B. \& Sen, N. (2011). Goal programming approach to tea industry of Barak Valley of Assam. Applied Mathematical Sciences, Vol. 5, No. 29, Pp. 1409-1419.

[21] Yahia-Berrouiguet \& Tissourassi (2015). Application of goal programming model for allocation of time and cost in project management. Yugoslav Journal of Operations Research, Vol. 25, No. 2, Pp. 283-289.

[22] Asllani, A., \& Lari, A. (2015). Using goal programming to increase the efficiency of marketing campaign. Journal of International and Interdisciplinary Business Research, Vol. 2, No.6, Pp. 53-68.

[23] Anggraeni, W., Vinarti, R. A., Tyasnurita, R., \& Permatasari, J. (2015). Production planning optimization using goal programming method in Habibah Busana. Journal of Advanced Management Science, Vol. 3, No. 4, Pp. 270-275. 